DOI: 10.2478/romneu-2013-0002

\title{
Posttraumatic extracerebral fluid collections
}

\author{
Danil Adam
}

Associate Professor, Department of Neurosurgery

"Carol Davila" University of Medicine and Pharmacy, Bucharest, Romania

Emergency Clinical "St. Pantelimon" Hospital, Șos. Pantelimon 240, Sect.2

Bucharest, Romania, e-mail: adam_danil@yahoo.com

\begin{abstract}
Objective: Posttraumatic extracerebral fluid collections represent a heterogeneous group of conditions that have different terminologies but the same clinical and imaging features, benefiting from the same therapeutic modalities.

Between hygroma/hydroma and serous meningitis there is only a topographic difference that has no influence regarding the therapeutic decision or the patient's outcome.

Posttraumatic extracerebral fluid collections present themselves as a unitary group which benefits from a unitary management, regardless the given terminologies. This 5 years study reviews the experience, treatment and outcome of patients with such conditions.
\end{abstract}

Method: A retrospective analysis of data collected from 46 patients with posttraumatic extracerebral fluid collections is presented. Asymptomatic patients or those with mild symptoms were conservatively treated $(n=27)$. Surgical evacuation was performed in cases of intracranial hypertension or neurological worsening $(\mathrm{n}=19)$.

Results: Clinical results are presented using the Glasgow Outcome Scale (GOS). Regarding the group of patients treated conservatively, good results were obtained
(GOS score 4 or 5 ) in $81.4 \%$ of the cases. Regarding the surgical treated group of patients, good results were obtained in $73.6 \%$ of the cases. The mortality rate was $18.5 \%$, and $26.3 \%$ respectively.

Conclusions: Although several different names can be found in literature, posttraumatic extracerebral fluid collections present themselves as a unitary group, with good outcome after conservative or surgical treatment. The therapeutic decision is made regarding the neurological status and the clinical evolution, which can be correlated with some imaging features.

Key words: hygroma, hydroma, serous meningitis, posttraumatic extracerebral fluid collections.

\section{Introduction}

Posttraumatic intracranial fluid collections have rarely been reported in the neurosurgical literature, although, with the implementation of modern imagery, their incidence has increased significantly. They represent a heterogeneous group of fluid collections which can be localized in the subdural space (hygroma, hydroma) or in the subarachnoid space (serous meningitis).

With a different pathophysiology, the two entities appear confounding on modern images.

This review focuses on subdural 
hygromas and serous meningitis and discusses clinical features and surgical treatment which are common to both conditions.

\section{Patients and methods}

All patients with posttraumatic extracerebral fluid collections diagnosed and treated in our institution were included in this study.

The patient's data was collected from hospital records and was retrospectively analyzed (Table 1).

The following clinical information was collected: age, sex, GOS score at admission, mechanism of injury, signs and symptoms and comorbidities.

The patients were investigated via CTscan at admission, which was repeated during hospitalization in case of worsening and also in postoperative period. There were taken into consideration: the size of the fluid collection, the midline shift and the coexisting brain injuries.

The treatment of the fluid collections was conservative for asymptomatic patients or for those with mild symptoms, with analgesics and hydration. It was administered in the neurosurgery ward or intensive care unit, depending on associated diseases requiring specific treatment.

Surgery was performed in cases of intracranial hypertension associated with altered consciousness, under general anesthesia by orotracheal intubation. The skull opening was performed with a trephine of 4.5 centimeters in diameter. After a star-shape opening of the dura mater and evacuation of the fluid under pressure, spontaneous and by suction, the dura mater was partially closed, the bone flap was restored and an epidural drain was left for 24-48 hours.
TABLE 1

Patients characteristics

\begin{tabular}{|c|c|c|c|}
\hline \multicolumn{4}{|c|}{ Total cases: 46} \\
\hline \multicolumn{2}{|c|}{ Age (years) } & No & $\%$ \\
\hline \multicolumn{2}{|c|}{ mean } & 68 & \\
\hline \multicolumn{2}{|c|}{ range } & $16-94$ & \\
\hline \multicolumn{4}{|c|}{ Sex } \\
\hline \multicolumn{2}{|l|}{ male } & 36 & 78.2 \\
\hline \multicolumn{2}{|c|}{ female } & 10 & 21.8 \\
\hline \multicolumn{4}{|c|}{ Mechanism of injury } \\
\hline \multicolumn{2}{|c|}{ fall from the same level } & 22 & 47.8 \\
\hline \multicolumn{2}{|c|}{ fall from another level } & 4 & 8.7 \\
\hline \multicolumn{2}{|c|}{ car accident } & 7 & 15.2 \\
\hline \multicolumn{2}{|c|}{ traumatic brain injury secondary to } & 2 & 4.3 \\
\hline \multicolumn{2}{|c|}{ unknown } & 11 & 23.9 \\
\hline \multicolumn{4}{|c|}{ Signs and symptoms at admission } \\
\hline \multicolumn{2}{|c|}{ headache and dizziness } & 20 & 43.4 \\
\hline \multicolumn{2}{|c|}{ psychomotor agitation } & 10 & 21.7 \\
\hline \multicolumn{2}{|c|}{ loss of consciousness for short } & 10 & 21.7 \\
\hline \multicolumn{2}{|c|}{ hemiparesis } & 8 & 17.3 \\
\hline \multicolumn{2}{|c|}{ dysarthria } & 7 & 15.2 \\
\hline \multicolumn{2}{|c|}{ temporo-spatial disorientation } & 7 & 15.2 \\
\hline \multicolumn{2}{|c|}{ balance disorders } & 6 & 13 \\
\hline \multicolumn{2}{|c|}{ otoragie } & 6 & 13 \\
\hline \multirow{4}{*}{ GCS } & $15-13$ & 19 & 41.3 \\
\hline & $12-19$ & 7 & 15.2 \\
\hline & $8-7$ & 4 & 8.7 \\
\hline & unspecified & 16 & 34.7 \\
\hline \multicolumn{4}{|c|}{ Comorobidities } \\
\hline \multicolumn{2}{|c|}{ arterial hypertension } & 16 & 34.7 \\
\hline \multicolumn{2}{|c|}{ chronic alcoholism and smoking } & 15 & 32.6 \\
\hline \multicolumn{2}{|c|}{ chronic ischemic heart disease } & 7 & 15.2 \\
\hline \multicolumn{2}{|c|}{ CPOD } & 5 & 10.8 \\
\hline \multicolumn{2}{|c|}{ ischemic stroke } & 5 & 10.8 \\
\hline CAS & & 3 & 6.5 \\
\hline diabet & & 4 & 8.7 \\
\hline cirrho & & 4 & 8.4 \\
\hline seizur & & 2 & 4.3 \\
\hline hemo & rhagic stroke & 1 & 2.2 \\
\hline
\end{tabular}

GCS-Glasgow Coma Scale, CPOD- Chronic

Pulmonary Obstructive Disease, CAS-Cerebral Athero-Sclerosis 
CT-scan was repeated in postoperative period and also before discharge in all cases, even if the patient was not operated.

The patient's clinical outcome was recorded on GOS scale 3 months later, when he came at a follow-up visit.

\section{Results}

Forty-six patients with posttraumatic extracerebral fluid collections were diagnosed and treated over a 5-year period.

Irrespective of subdural or subarachnoid topography of fluid, the patients had similar clinical and radiological features. There were patients treated conservative (Group A) and patients treated by surgery (Group B). There were no differences in treatment modalities regarding the topography of fluid.

\section{Demographics}

There were a total of 46 patients. There were 36 males and 10 females. The youngest patient was 16 years old and the oldest was 94 years old. The average age of patients with posttraumatic extracerebral fluid collections was 68 years.

The distribution per decade of age is presented in Table 2.

\section{Radiological data}

Brain CT scans were obtained from patients preoperative and postoperative.

CT-scan revealed a simple fluid collection, not associated with brain injuries, in 6 cases.

Brain injuries associated with fluid collection, shown on the CT scan are presented in table 3 and in figures 1-5.

TABLE 3

Brain injuries associated with fluid collections

\begin{tabular}{|lcc|}
\hline Associated lessions & No. & $\%$ \\
hemorrhagic contusions & 25 & 54.3 \\
skull fracture & 12 & 26 \\
cerebral atrophy & 11 & 23.9 \\
epidural hematoma & 2 & 4.3 \\
intraparenchymatous & 4 & 8.7 \\
hematoma & & \\
subdural hematoma & & \\
\multicolumn{1}{c}{ acute } & 3 & 6.5 \\
\multicolumn{1}{c}{ chronic } & 9 & 19.6 \\
\hline
\end{tabular}

TABLE 2

Distribution per decade of age

\begin{tabular}{|lcccccccccc|}
\hline & $\mathbf{1 0 - 1 9}$ & $\mathbf{2 0 - 2 9}$ & $\mathbf{3 0 - 3 9}$ & $\mathbf{4 0 - 4 9}$ & $\mathbf{5 0 - 5 9}$ & $\mathbf{6 0 - 6 9}$ & $\mathbf{7 0 - 7 9}$ & $\mathbf{8 0 - 8 9}$ & $\mathbf{9 0 - 9 9}$ & Total \\
$\mathbf{F}$ & 1 & - & - & - & - & 2 & 5 & 2 & - & 10 \\
$\mathbf{M}$ & - & - & 3 & 2 & 4 & 7 & 13 & 6 & 1 & 36 \\
Total & 1 & - & 3 & 2 & 4 & 9 & 18 & 8 & 1 & 46 \\
\hline
\end{tabular}



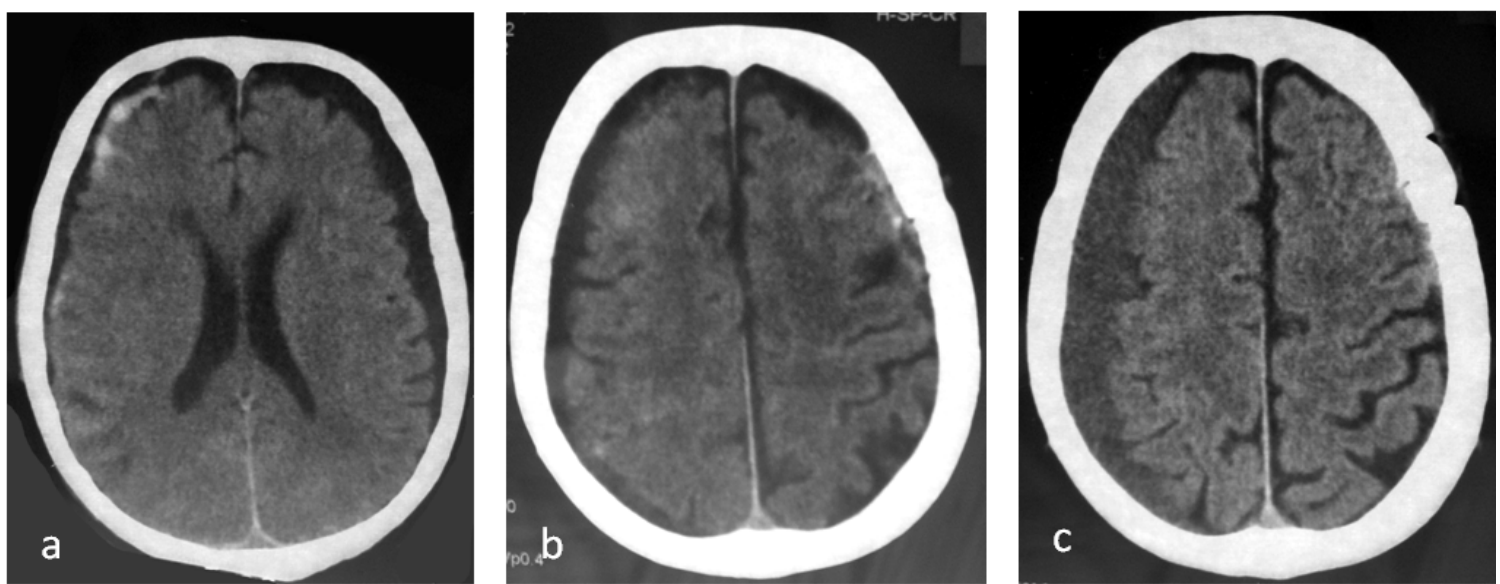

Figure 1 Male, 74, with head trauma caused by accidental 2 meter fall on the stairs, with bilateral hygroma right $>$ left (a). A week after the evacuation of the hygroma in the left side a growth of extracerebral collection in the right side is observed (b); after 3 weeks it transformed into a chronic subdural hematoma $18 \mathrm{~mm}$ thick; the midline shifted $4 \mathrm{~mm}$ towards the left side (c). Note that the hygroma from the right side was associated with hemorrhagic cortical contusions (complex hygroma).

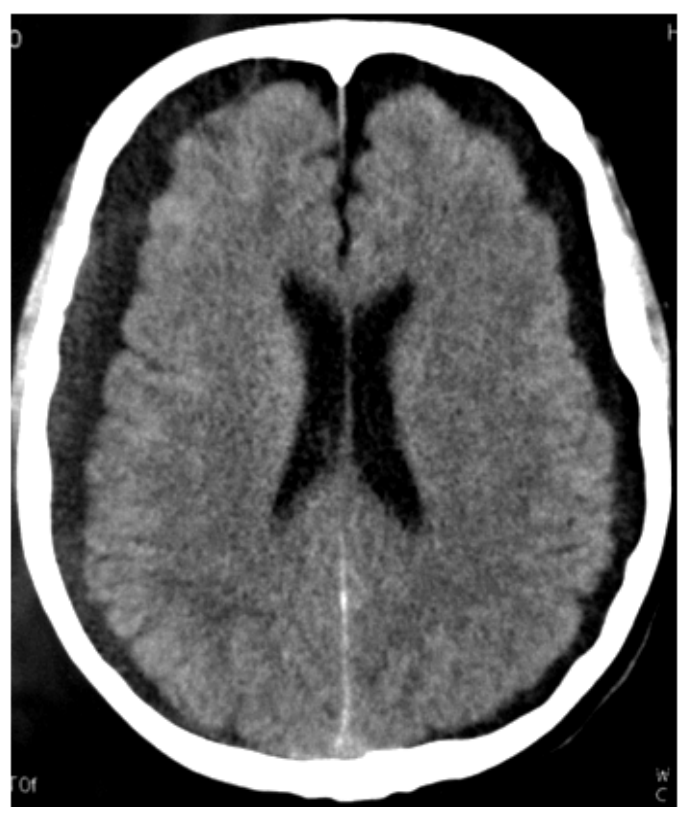

Figure 2 Male, 60, with head trauma caused by accidental fall from the same level. Cerebral CT scan highlights extracerebral fluid collection, bilateral, 12 $\mathrm{mm}$ on the right side and $8 \mathrm{~mm}$ on the left side, without associated cerebral damage (simple bilateral hygroma).
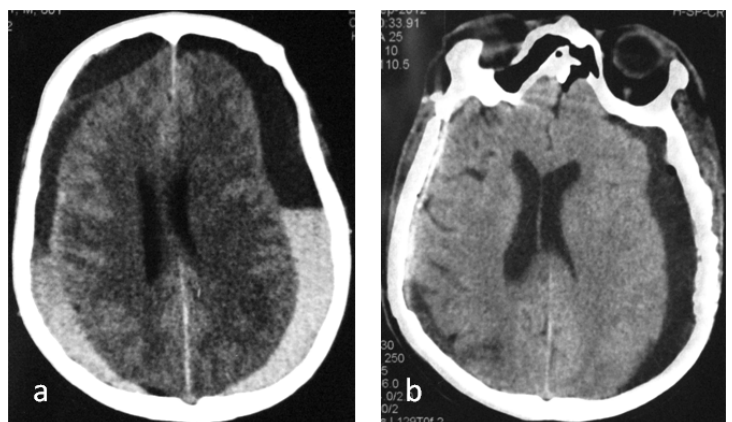

Figure 3 Male, 81, with head trauma caused by fall from the same level. Cerebral CT scan in axial plane highlights: bilateral subdural hematoma (a). Two weeks after surgery it is observed that the collection on the right side disappeared and that a hygroma developed on the left side (b).
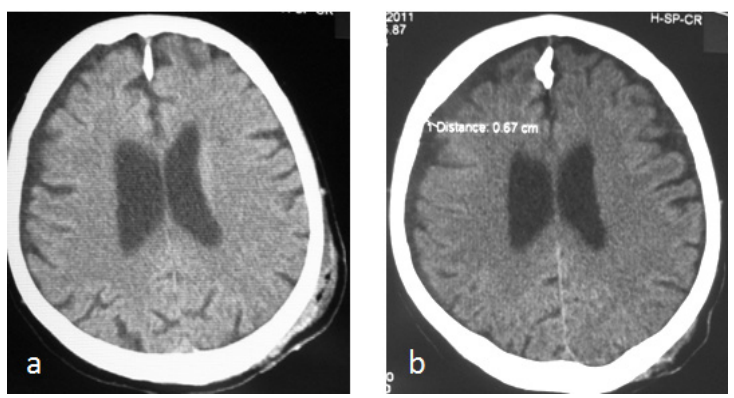

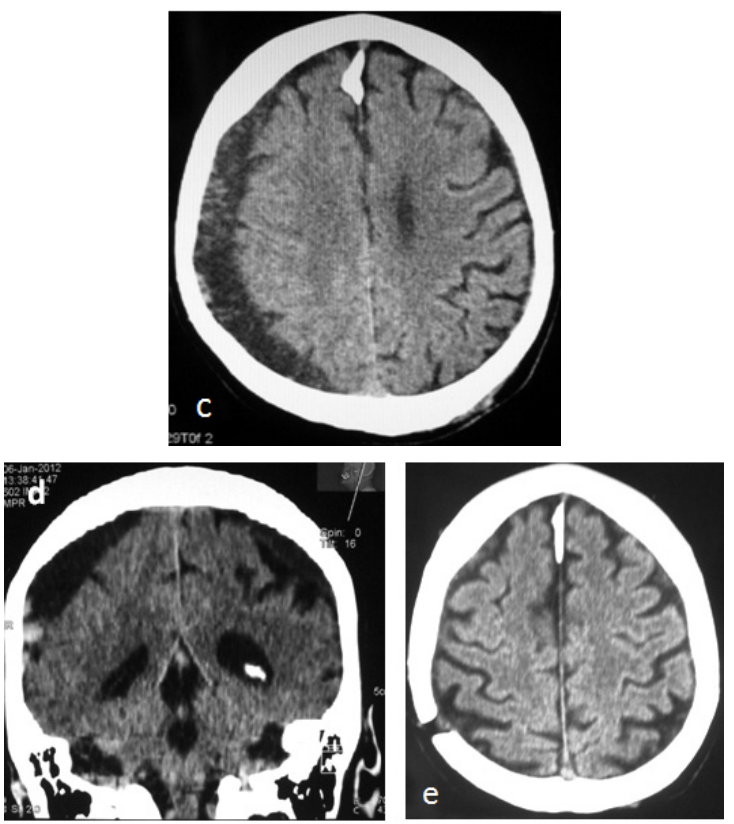

Figure 4 Male, 73, with head trauma caused by fall from the same level. Cerebral CT scan at admission highlights a small extracerebral fluid collection on the right side (a), which increased in dimensions after a week (b); two weeks later cerebral CT in axial and coronal planes highlights a right-sided chronic subdural hematoma and on the left side a chronic

SDH formed from fluid associated with an underlying hygroma with clear-colored fluid during surgery (c) and (d). Postoperatory CT scan shows brain expansion after the surgical evacuation of blood and fluid collections (e).
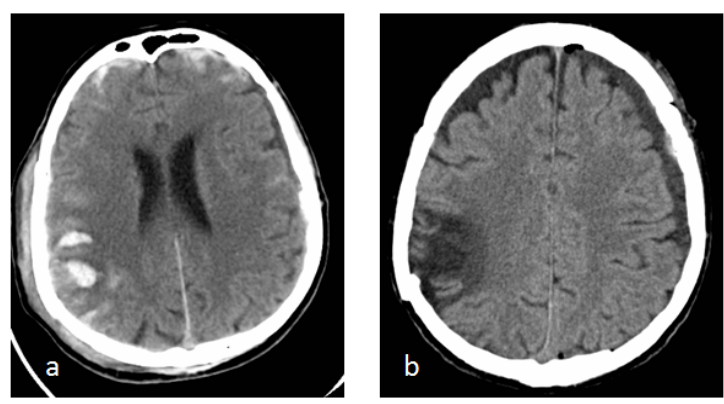

Figure 5 A 16 year old patient with head trauma caused by car accident. CT scan in axial plane (a), shows multiple cerebral concussions at admission: bifrontal and temporoparietal right-sided underlying a cranial fracture. After 3 weeks the cerebral CT scan in axial plane shows a development of left-sided frontoparietal and right sided frontal, $4 \mathrm{~mm}$ thick extracerebral fluid collections.
The fluid collection was unilateral in 21 cases and bilateral in 25 cases.

The fluid collection development appeared at a variable interval from the traumatic injury, between 24 hours and 50 days.

The relationship between the size of the fluid collection, midline shift, applied treatment and outcome was followed. Regarding the patients with conservative treatment, the size of the fluid collection in the axial plane was between 4 and $14 \mathrm{~mm}$ and the midline shift oscillated between 0 and $7 \mathrm{~mm}$. There were 8 patients with 0 $\mathrm{mm}$ midline shift, 4 patients with $3 \mathrm{~mm}$ midline shift, 7 patients with $4 \mathrm{~mm}$ midline shift and 1 patient with $7 \mathrm{~mm}$ midline shift. In 10 cases, the midline shift was not mentioned. In 6 cases, the size of the fluid collection was not mentioned. In 13 cases regarding the group of patients treated conservatively, the hygroma was bilateral. The medical treatment was applied to 27 patients.

Regarding the patients who underwent surgery, the size of the fluid collection in the axial plane varied between 6 and $18 \mathrm{~mm}$ and the midline shift varied between 0 and $4 \mathrm{~mm}$, but in most cases the midline shift was not mentioned. The decision to operate was made when neurological deterioration occurred. In 12 cases regarding the group of patients who underwent surgery, the hygroma was bilateral. 19 patients underwent surgery. Among these, in 12 patients the fluid evacuated after dura incision was citrine, in 2 cases it was clear and in 5 cases the color was not mentioned. In 7 patients it was observed the existence of a vascularised parietal subdural membrane, with a variable thickness, about $1 \mathrm{~mm}$, unaccompanied by the existence of a visceral membrane. 


\section{Clinical outcome}

The outcome regarding patients with intracranial fluid collections is presented in Table 4.

TABLE 4

Outcome according to GOS (Glasgow Outcome Scale)

\begin{tabular}{|l|cccc|}
\hline GOS & \multicolumn{2}{|c|}{ Group A } & \multicolumn{2}{c|}{ Group B } \\
\cline { 2 - 5 } characteristics & No. & \% & No. & \% \\
\hline Good recovery & 19 & 70.3 & 12 & 63.1 \\
Moderate & 3 & 11.1 & 2 & 10.5 \\
disability & & & & \\
Severe disability & - & & - & \\
Vegetative state & - & & - & \\
Death & 5 & 18.5 & 5 & 26.3 \\
\hline
\end{tabular}

The causes of death, regarding the group of patients treated conservatively, were: liver failure associated with decompensated diabetes (1 case), exacerbation of chronic kidney disease (1 case), bronchopneumonia (1 case), and severe traumatic brain injury (2 cases).

In the surgical group, 2 patients died because of pulmonary complications (bronchopneumonia), 1 patient died because of an ischemic stroke and 2 died because of the association of the hygroma and a subdural hematoma.

\section{Discussions}

There is a terminological ambiguity regarding the designation of extracerebral fluid collections.

Subdural collections of fluid other than blood are referred to as sudural hygromas or hydromas.

The term subdural hydroma was first reported by Mayo [1] in 1894, but subsequently evolved into subdural hygroma or subdural effusion. Described initially by Payr [2] in 1916, the hygroma was separated as a nosologic entity under this name by Dandy [3] in 1932. There is considerable confusion about these entities. In Greek "hygros" means "wet".

The hygroma represents a fluid collection circumscribed in the subdural space, containing a xantocorm liquid which is bounded by a neomembrane [4].

In the case of hydroma, the liquid is clear and the membrane is missing.

The serous meningitis is a circumscribed or diffuse accumulation of fluid located in the subarachnoid space. It is often confused with external hydrocephalus.

Between serous meningitis and hygroma/ hydroma there is only a difference of topography.

Although the fluid collections were identified at almost any age, there is a net movement of their occurrence over the age of 60 . The youngest patient in our series was 16 years old and he was involved in a car accident. Mayo reported the case of o 12 year-old boy who, secondary to head trauma, developed o fluid collection overlying the left cerebral hemisphere.

\section{The mechanism}

As well as the chronic subdural hematoma (SDH), the hygroma develops in the subdural space. Chronic SDH derives from a subdural hygroma and vice versa.

The cerebrospinal fluid leaks through an arachnoid breach detaching the dural border cells layer and produces a subdural collection. Naffziger [5] discovered an arachnoid membrane rupture in 1924.

As well as the chronic SDH, hygroma has a membrane. It forms as a result of an inflammatory reaction which follows the initial injury. The inflammatory reaction is characterized by cellular proliferation in the 
dural border layer with formation of granulation tissue along with collagen fibers and blood vessels. The neo-formation membrane facing the arachnoid mater is avascular. The parietal side contains blood vessels. The subdural hygroma may evolve in a chronic SDH. Also the hygroma may represent the final stage of chronic $\mathrm{SDH}$ when the fluid is watery, contains no blood and is similar to the cerebrospinal fluid (CSF). In rare cases hygroma coexists with chronic SDH.

Serous meningitis is a fluid, compressive subarachnoid collection which causes intracranial hypertension. It is common in elderly adults and it develops in cases of external hydrocephalus and cerebral atrophy and it is bilateral in the majority of cases. The fluid collection fills the space between the atrophic brain and the arachnoid.

The tearing of the arachnoid is generated by kinetic energy and shear stress caused by the trauma. The hygroma increases in volume due to a valve mechanism in the arachnoid breach which allows unidirectional CSF flow from the subarachnoid space towards the outside when cough or sneeze occur or as a result of arterial pulse.

The interface tear between the dura mater and the arachnoid can also be a result of surgery [6]. This explains how hygroma can be secondary to a decompressive craniotomy or to a chronic subdural hematoma evacuation.

According to Schneider [7], the subdural fluid is a plasma exudation resulting from high permeability of some blood vessels from the dura mater.

The trauma was mentioned in all of the cases in our series of 46 patients while the type of trauma was specified in $75 \%$ of the patients. The most frequent head traumas were those with the same level fall $(n=22)$ occurring in elderly patients. In a single case the trauma was associated with a blood disorder secondary to thrombocytopenia. In elderly persons hygroma as well as chronic SDH may develop after a minor head trauma.

\section{Occurrence}

Fluid collections after head trauma have an occurrence of 5 to $21 \%[8,9,10]$ reaching $25-30 \%$ secondary to a decompressive craniotomy [11, 12].

\section{Signs and symptoms}

The clinical picture of intracranial fluid collection is nonspecific. The signs and symptoms can be assigned to the trauma and not to a specific clinical entity.

Liquid collections occurred both as a result of minor head trauma and also secondary to severe head trauma.

In addition to cases with mild symptoms there were also cases presenting symptoms which progressed to worsening, the headache increasing in intensity. Moreover, some patients presented psychomotor agitation $(21,7 \%)$ or were confused, disoriented (15\%).

Medical history such as cerebral arteriosclerosis or stroke were factors in increased brain atrophy regarding the elderly; in addition we can observe that smoking and alcohol consumption are also risk factors, each found in $32 \%$ of patients with intracranial fluid collections.

\section{Radiological investigations}

Subdural fluid collections can be routinely detected through computerized tomography scans.

The CT-scan revealed the extracerebral collection as hypodense, with a density similar to CSF, uni- or bilateral. In our 
series of patients we came across bilateral collections in $54 \%$ of the cases.

In the axial plane the dimensions of the fluid collections varied between 4 and 15 $\mathrm{mm}$. However, the midline shift did not increase accordingly - either as a result of the bilateral collection or because it developed overlying an atrophic brain in elderly persons.

The either surgical or conservative treatment was performed according to clinical status at admission or to its following evolution, as well as according to the axial dimensions of the fluid collections; the midline shift was a minor contributing factor for surgical treatment.

The majority of the patients were treated conservatively and showed improvement as a result of hydration and neuroprotective treatment. Hydration along with intracranial hyper-tension allowed the fluid collections to resolve spontaneously along with brain expansion. $41 \%$ of the patients underwent surgical evacuation of the fluid collections.

The surgery was a trephine craniotomy $4,5 \mathrm{~cm}$ in diameter, star shaped section of the dura mater and under pressure evacuation of the subdural fluid.

CT-scan images revealed different aspects:

- complex unilateral hygroma

- simple bilateral hygroma

- complex bilateral hygroma which after the evacuation of fluid collections from one side became a chronic subdural hematoma of the opposite side

- chronic bilateral subdural hematoma; a unilateral hygroma occurred after its evacuation

- initially small hygroma which became thicker - SDH occurred on one side and on the opposite side chronic SDH was associated with an underlying hygroma

The CT-scans highlighted the extracerebral fluid collection/collections and any potential brain damage but they did not differentiate between a hygroma, hydroma, serous meningitis or the final phase of a chronic SDH.

\section{Differential diagnosis}

There have been attempts to differentiate subdural hygroma from chronic SDH based upon fluid characteristics.

The hygroma fluid contains prealbumin found in CSF but not in subdural hematoma [13]. The hygroma fluid has a content of less than $106 \mathrm{RBCs} / \mathrm{ml}$ while in the subdural hematoma the quantity of RBCs $/ \mathrm{ml}$ (Red Blood Cells $/ \mathrm{ml}$ ) is bigger [14].

\section{Management and outcome}

The majority of the extracerebral subdural collections is asymptomatic and progressively or spontaneously disappears. Regarding post decompressive craniotomy hygroma, Aarabi et al [12] observed that the hygromas appear in the first postoperatory week; they reach maximum volume in the 4th week and disappear around the 17th week. As far as the symptomatic hygromas are concerned, the clinical improvement advocates in favor of conservative treatment.

Patients with complex hygromas require clinical and imagistic follow-ups and a symptomatic treatment of underlying cerebral damage.

Operative decision making is based upon change in the patient's state of consciousness associated with growth of hygroma. 
Mass effect defined by a midline shift bigger than $5 \mathrm{~mm}$ is not always a good indicator of surgery; hygromas are of high occurrence among the elderly, along with cerebral atrophy and external hydrocephalus. A cerebral damage with an edema may create a false impression of subdural fluid collection on the initial CTscans.

One of the patients in our series had a $7 \mathrm{~mm}$ shift and evolved favorably under conservative treatment. Furthermore, among the group that underwent surgery there were patients with a shift smaller than $5 \mathrm{~mm}$ and required surgery.

The surgery was in all cases a trephine craniotomy with a diameter of $4.5 \mathrm{~cm}$. This allowed a better view of the operative field and an accurate hemostasis of the dura and parietal membrane.

The general mortality of the entire series was $21.7 \%$ - $18.5 \%$ in the medical group and $26.3 \%$ in the surgical group (figure 6).

Causes of death were the pre-existing medical conditions that were uncompensated by occurrence of pulmonary complications in elderly patients or cerebral ischemic complications. Two patients who were medically treated deceased after suffering severe head trauma.

Mortality is more related to additional brain lesions or to systemic complications during hospitalization than to hygroma itself.

\section{Conclusions}

Posttraumatic extracerebral fluid collections can be generally found under varied terminologies. There are no differentiating imagistic aspects and no characteristic symptomatology for hygroma, hydroma or serous meningitis; thus, a terminological uniformity is necessary in the form of extracerebral fluid collections or plainly, hygroma.

Hygroma diagnosis is performed through CT-scans of all of the patients with craniocerebral trauma at admission and depending on the evolution, scans are repeated during hospitalization and at discharge.

The majority of patients whose CTscans show extracerebral fluid collections are asymptomatic and the fluid collections are eventually spontaneously resolved.

Surgery is indicated when the clinical picture shows neurological damage.

Evacuation of subdural fluid collections can be performed through various methods. We prefer trephine craniotomy which allows a better view and an accurate hemostasis.

Mortality is in most cases caused by associated cerebral damage or systemic complications. 


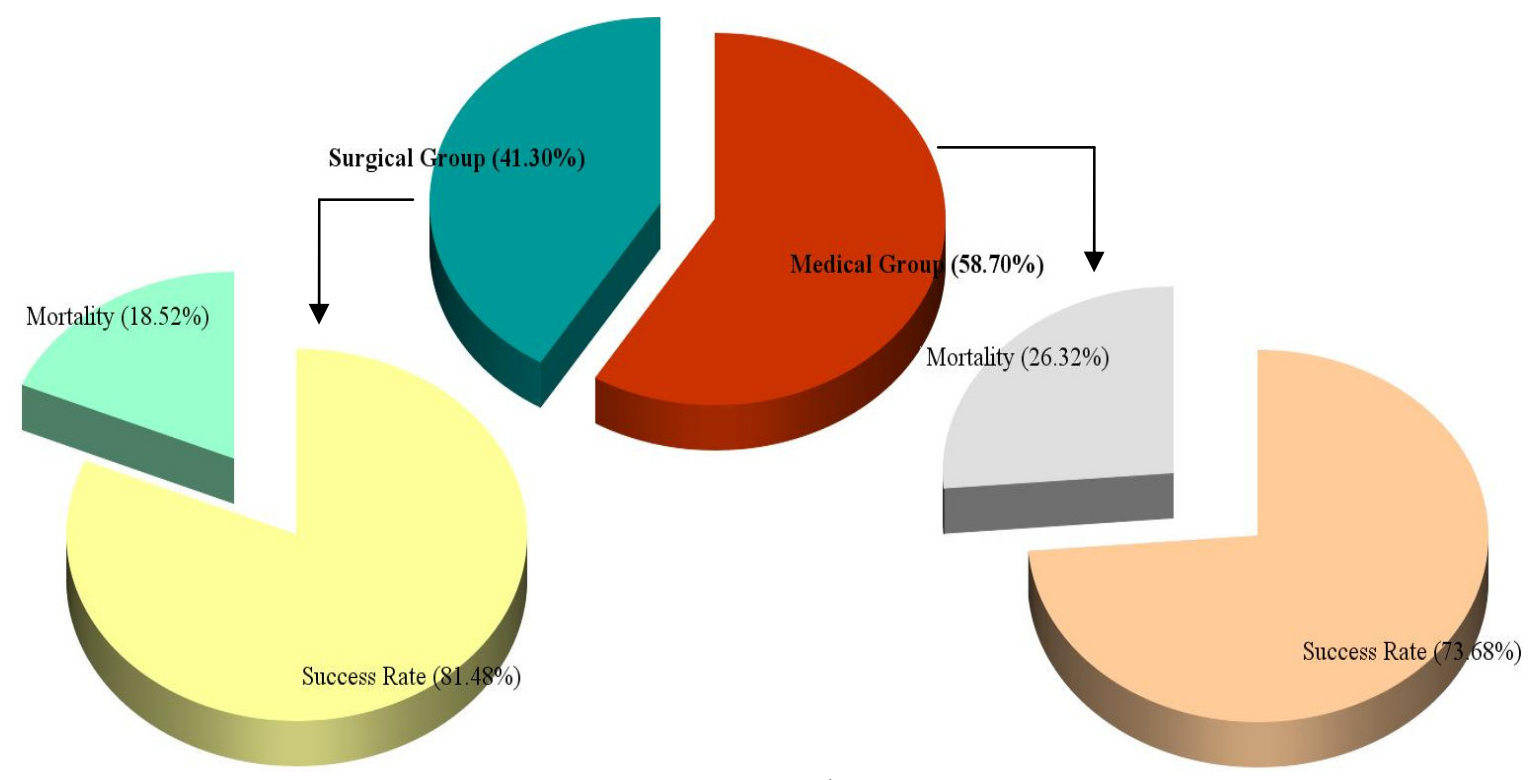

Figure 6 Mortality rate

\section{References}

1. Mayo CH. A brain cyst: The results injury causing aphasia, hemiplegia, etc. Evacuation; complete recovery. NY Med J. 1894; 59:434,.

2. Payr E. Meningitis serose bei und nach Schadel Verlezungen (traumatica). Med Klin. 1916; 12: 841-846. 3. Dandy WE. Chronic subdural hydroma and serous meningitis (pachymeningitis serosa; localise external hydrocephalus). In: Lewis D (ed): Practice of Surgery, Hagerstom, Maryland W. F. Prior 1932, p. 306-314.

4.Arseni C, Oprescu I. Neurotraumatologie, Editura Didactică si Pedagogică, Bucuresti 1983, p. 163.

5. Naffziger HC. Subdural fluid accumulations following head injury. JAMA 1924; 82: 1751-1752.

6. Pardes I, Cicuendes M, Delgado AM, et al. Normal pressure subdural hygroma with mass effect as a complication of decompressive craniectomy. Surg Neurol Int 2011; 2:88.

7. Schneider RC. Head injuries in infancy and childhood. Surg. Clin. North Arm. 1961; 41: 1255.

8. Born JD, Hans P, Flandroy P, et al. Incidence and treatment of subdural hygroma in severe head injuries.
Acta Anaesthesiol Belg. 1984; 84: 174-182.

9. Ohno K, Suzuki R, Masaoka H, et al. Chronic sundural haematoma preceded by persistent traumatic subdural fluid collection. J Neurol Neurosurg Psychiatry 1987; 50: 1694-1697.

10. Lee KS, Bae WK HG, Yun IG. The fate of traumatic subdural hygroma in serial computed tomographic scans. J. Korean Med Sci 2000; 15: 560-568.

11. Yang XF, Wen L., Shen F, et al. Surgical complications secondary to decompressive craniectomy in patients with a head injury: a series of 108 consecutive cases. Acta Neurochir (Wien) 2008; 150: 1241-1247.

12. Aarabi B, Chesler D, Maulucci C, et al. Dynamics of subdural hygroma following decompressive craniectomy: a comparative study. Neurosurg Focus 2009; 26(6): E8.

13. Greenberg SM. Handbook of Neurosurgery, Thrid Edition, Greenberg Graphics, Inc. Lakeland, Florida, 1993, p. 546.

14. Samandouras G. The neurosurgeon's handbook. Oxford University Press Inc, New York, 2010, p. 689. 